\title{
The Effect of Billet Wall Thickness on the Rotary Compression Process for Hollow Parts
}

\author{
Janusz Tomczak* - Tomasz Bulzak - Zbigniew Pater \\ Lublin University of Technology, Poland
}

This paper presents the numerical and experimental results of investigating the effect of geometrical parameters of tubular specimens on the rotary compression process for hollow axisymmetric shafts with central necking, and their accuracy. The numerical investigation was performed using the finite element method (FEM) with the Simufact Forming simulation software suite. The simulations investigated the effect of tubular specimen wall thickness on the kinematics of metal flow and the geometry of produced shafts. The results were validated in experimental tests using a forging machine designed specifically for this purpose.

Keywords: rotary compression, hollow parts, finite element method, experiment

Highlights

- A new method for producing hollow parts is presented.

- The relationship between the wall thickness of a tubular specimen and rotary compression is investigated.

- The effect of wall thickness on the rotary compression process is studied both numerically and experimentally.

- The distributions of effective strain and temperature are determined by the finite element method.

- The variations in length and thickness of the work piece wall are determined, too.

\section{INTRODUCTION}

Hollow parts, such as stepped axes, shafts, tubes, rings and many other axisymmetric parts, have a variety of applications, from automotive and aircraft structures to household appliances [1]. Due to their being highly in demand, hollow parts with finishing allowances are increasingly produced by metal-forming processes [2]. Compared to machining, metal-forming methods for producing hollow parts ensure reduced material consumption as well as enhanced strength properties of parts (fibre continuity is maintained). It should, however, be noted that the majority of current metalforming processes for hollow parts are significantly complicated [3]. As a result, it is expensive to run them and, hence, to implement new technologies [4]. For this reason, new highly-efficient methods for forming hollow parts are being developed to ensure the production of parts with the required geometry and strength at the lowest costs possible.

The innovative methods for forming hollow parts from tubular billets include hydroforming and the RoProFlex method developed by the Institute of Forming Technology and Lightweight Construction (IUL) in Dortmund, Germany. The hydroforming technique is used to produce hollow complexshaped parts that cannot be produced as monolithic components by other techniques. Parts produced by hydroforming are applied in automotive and aircraft industries. The main disadvantages of hydroforming include the high costs of tools, the slow speed of the process, and a lack of information about the process and tool design [5]. The RoProFlex method can be used to form any cross sections of tubes and sections along their longitudinal axis. Thanks to the numerical control of tool motion, this method can be used to produce parts with limitless variations of their cross sectional geometry. The RoProFlex method is mainly applied to produce light car parts, precise elements such as gears and helical rotors, as well as medical implants [6].

Rotary compression can be used to produce axisymmetric hollow shafts, including toothed shafts with straight, helical and herringbone teeth, as well as worms. In contrast to hydroforming and RoProFlex, this technique can be employed to form parts with thicker walls. The machine for rotary compression has a very simple design. The disadvantage of rotary compression is that this technique cannot be used to produce non-axisymmetric parts that can, however, be produced by hydroforming and RoProFlex.

One of the innovative methods for forming axisymmetric hollow parts is rotary compression shown in Fig. 1.

Rotary compression is a metal-forming operation based on the technique of cross rolling with three rolls, in which the tools not only rotate, but they also simultaneously travel towards the axis of the work piece. In this process, sections of commercial tubes or sleeves are rotated and deformed to reduce the diameter of steps. Compared to current techniques for manufacturing hollow shafts, rotary compression 
offers numerous benefits, including lower forming forces, reduced material consumption, increased strength properties of produced parts, enhanced process efficiency, lower implementation and production costs, as well as simple design of the process and the possibility of its automation. Given the above benefits, it can be claimed that rotary compression can be applied in the industry. Therefore, the present investigation undertakes both theoretical and experimental analyses to develop relationships between the individual parameters of the rotary compression process for hollow parts and product quality.
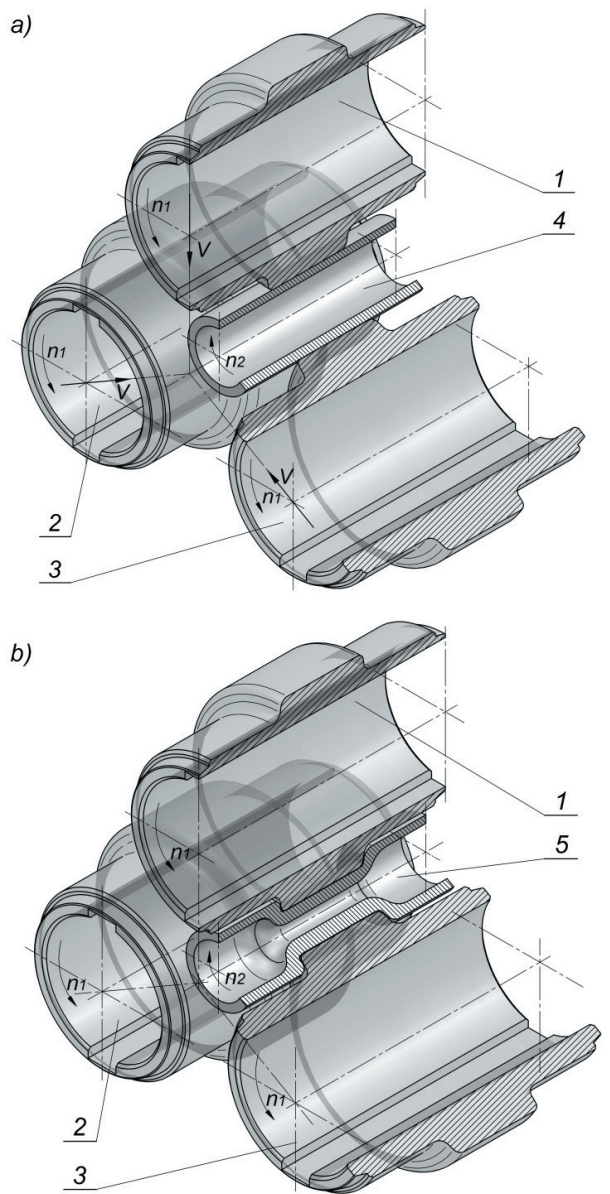

Fig. 1. Schematic diagram of the rotary compression process for hollow shafts; a) start of the process, and b) end of the process; 1, 2 and 3 working rolls, 4 specimen, 5 finished part

\section{FEM MODELLING OF THE ROTARY COMPRESSION PROCESS}

The present investigation mainly deals with presenting the effect of wall thickness of tubular specimens on rotary compression and product quality in the course of forming hollow necking in the centre of a shaft. The geometry of the work piece is determined by the reduction ratio $(\delta=D / d)$ of the step (where: $D$ is the outer diameter of the tubular specimen, $d$ is the outer diameter of the step), which was set to 1.5 and of the initial wall thickness to of the tubular specimen set to 3, 5, 7, 9 and $11 \mathrm{~mm}$, respectively. The shape and dimensions of the tubular specimen and of the desired final product are shown in Fig. 2.

First, the rotary compression process for a hollow shaft was modelled numerically. The computations were performed by the finite element method (FEM) using the Simufact Forming simulation software suite version 12.0 , which the authors had previously used to investigate rotary processes for forming metals.

The geometrical model of the process developed for the purposes of the numerical analysis was identical to the schematic design of rotary compression shown in Fig. 1. The model consisted of three identical forming rolls and a tubular specimen (a C45 steel tube that had an outer diameter, $D$, of $42.4 \mathrm{~mm}$, a wall thickness, $t_{o}$ of $3,5,7,9,11 \mathrm{~mm}$ and a length, $L$, of $120 \mathrm{~mm}$ ), modelled by means of first-order eight-node hexahedral elements. The model of C45 steel used in the computations was taken from the library database of the software used and is defined by:

$$
\begin{gathered}
\left.\sigma_{p}=2859.85 \times e^{(-0.00312548 \times T)}\right) \times \varepsilon^{(0.000044662 \times T-0.101268)} \times \\
\times e^{\frac{(-0.000027256 \times T+0.000818308)}{\varepsilon}} \times \dot{\varepsilon}^{(0.000151151 \times T-0.00274856)},
\end{gathered}
$$

where $T$ is the temperature (ranging from 700 to 1250 $\left.{ }^{\circ} \mathrm{C}\right), \varepsilon$ is the effective strain, $\dot{\varepsilon}$ is the strain rate.

At the initial stage of the process, the tubular specimen was heated throughout its volume to a temperature of $1150{ }^{\circ} \mathrm{C}$, while the temperature of the tools was maintained constant at $100{ }^{\circ} \mathrm{C}$. In the experiments, the tools were heated to a temperature of $100{ }^{\circ} \mathrm{C}$ with a gas burner. The temperature of the tools was monitored using a Flir T425 thermal imaging camera. The rolls were rotated in the same direction at a constant velocity $n=36 \mathrm{rpm}$, and simultaneously travelled relative to the axis of the work piece at a constant velocity $v=2.0 \mathrm{~mm} / \mathrm{s}$. The friction factor $m$ on the material-tool contact was set to 1 [7], the material-tools heat transfer coefficient was $30 \mathrm{~kW} / \mathrm{m}^{2} \mathrm{~K}$ [8], while the heat transfer coefficient between the material and the environment was set to $0.35 \mathrm{~kW} / \mathrm{m}^{2} \mathrm{~K}$ [9]. 


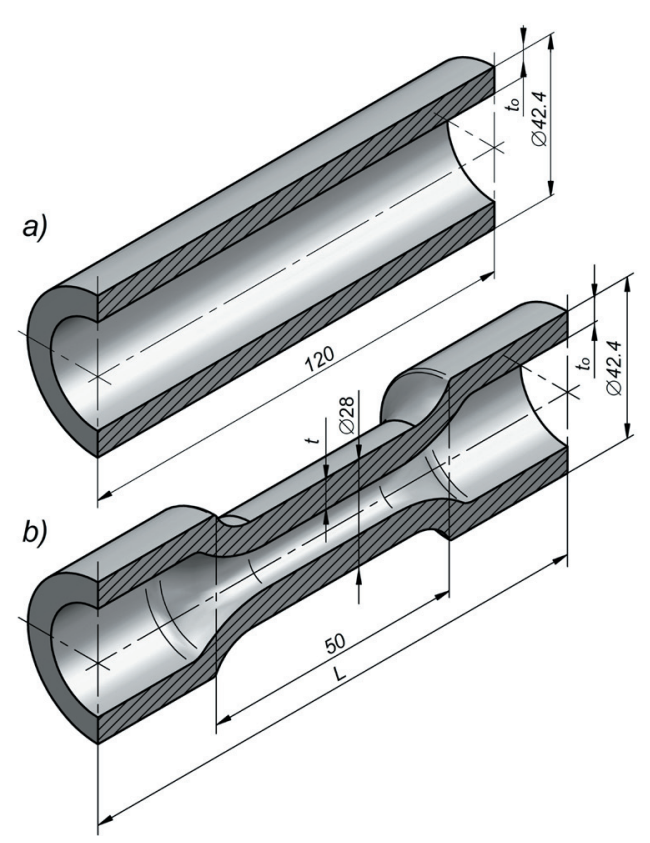

Fig. 2. Shape and dimensions of a) tubular specimen, b) hollow shaft

During the process, the rotating tools form necking in the centre of the shaft, which is accompanied by rapid metal flow radial to the axis of the work piece, thus leading to an increase in the thickness of the walls in this region. Furthermore, it can be noted that the material travels along the axis of the tubular specimen, which results in an increase in the work piece length. At the final stage of the process (i.e. sizing), the tools only perform a rotational motion, removing inaccuracies produced in the previous stages of the process. The FEMdetermined geometry of hollow shafts and the effective strains for tubular specimens with different initial thickness of the wall are illustrated in Fig. 3. It is characteristic of the strains that they are distributed in a non-uniform manner. In all examined cases, the highest strains are concentrated on the outer surface of the work piece wall. The closer it is to the internal wall, the smaller the strains are. Such a distribution of strains is typical of rotary metal-forming processes and can be attributed to the kinematics of tool and work piece motion. Considerable slipping between the material being formed and the tools, as a result of which additional deformations are generated in the circumferential direction (by friction forces) on the work piece surface, can be observed. That increasing the initial thickness of the tubular specimen wall not only leads to an increase in the maximum strains, but to a greater non-uniformity of these strains can also be observed. This results from higher resistances to flow of the material during the forming of tubular specimens with thicker walls.

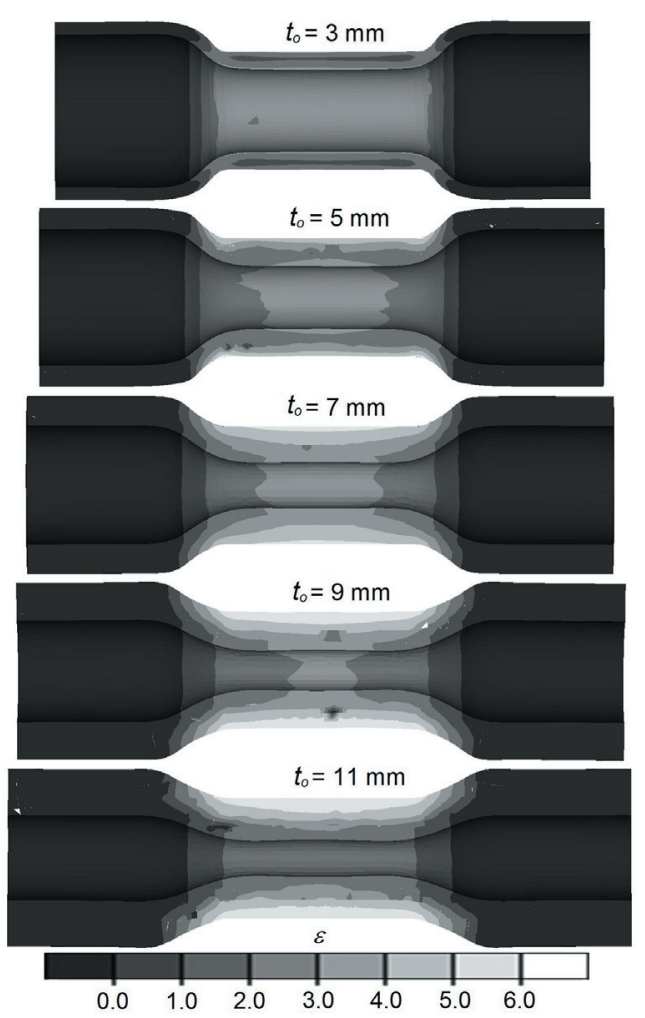

Fig. 3. FEM-calculated distributions of effective strains in the final stage of the process for tubular specimens with various wall thicknesses

The heat capacity of the hollow tubular specimens is much lower than that of the solid tubular specimens, which means that the material cools faster, thus hindering the rotary compression process. For this reason, the present investigation involved determining the effect of tubular specimen wall thickness on temperature and its distribution. Fig. 4 shows the temperature distribution maps that were calculated by the FEM in the final stage of rotary compression for tubular specimens with various wall thicknesses.

As predicted, the distributions of the temperatures are not uniform; the highest drops in the temperature can be observed in the region of the necking being formed. The non-uniform distribution pattern of the temperature in the region of the necking being formed results from the nature of the rotary compression process. During this process, the outside layers of the work piece are in contact with the colder tools, thus carrying heat away to the tools. In contrast, the inside layers of the work piece lose heat mainly due to heat exchange with the environment. The non- 
uniform temperature distribution in the region of the necking being formed results from that fact that heat is transferred at a more rapid rate to the tools than to the environment. The amount of heat transferred from the work piece to the tools and the environment is determined by the heat transfer coefficients, the magnitudes of which are given in the section describing the numerical model.

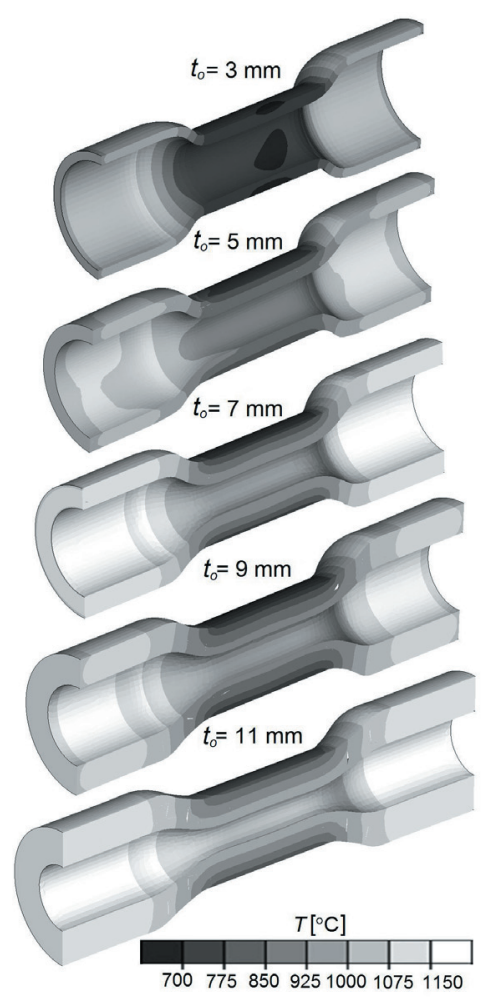

Fig. 4. FEM-calculated distributions of the temperature in the final stage of the process for tubular specimens with various wall thicknesses

The design of this forming process assumed that the external steps would not be in contact with the tools. As a result, they did not undergo deformation and their temperature was relatively uniform and high (above $1000{ }^{\circ} \mathrm{C}$ ). Examining the distribution of the temperature in the zone where the tools have a direct impact on the tubular specimen, it can be observed that the highest drop in the temperature occurs when the tubular specimens with small wall thickness are used (low heat capacity of the tubular specimen). In addition, the forming of tubular specimens with relatively thin walls leads to the cooling of the material, practically over the entire section of the reduced step, and the temperature distributions in the reduction area are quasi-uniform. In contrast, the distributions of the temperature for tubular specimens with thicker walls differ: the temperature drops locally (on the surface). The farther it is from the surface layers that directly contact the tools, the higher the temperature. In effect, such a temperature distribution pattern enables the heat to flow from the inner to outer surfaces of the work piece, so that the temperature of the step is high enough for a relatively long time for the hot forming of this step. The rotary compression of tubular specimens with small thickness of their walls is, however, harder to perform, as the rapid and excessive cooling of the material across the entire section of the step being formed leads to a decrease in workability of the material and an increase in resistances to forming.

\section{EXPERIMENTAL TESTS OF THE ROTARY COMPRESSION PROCESS FOR PRODUCING HOLLOW SHAFTS}

The numerical results were verified experimentally under laboratory conditions. To perform the experiments, we used a forging machine as shown in Fig. 5. With this machine, the process can be run in accordance with the pattern of the numerical simulations. Force and kinematics parameters of the rotary compression process were measured with a measuring system comprised of the following elements: a torque transducer to digitally record torque during forming; a displacement transducer to record velocity and position of the tools; pressure transducers to measure pressure in order to determine the force of the tools acting on the work piece.

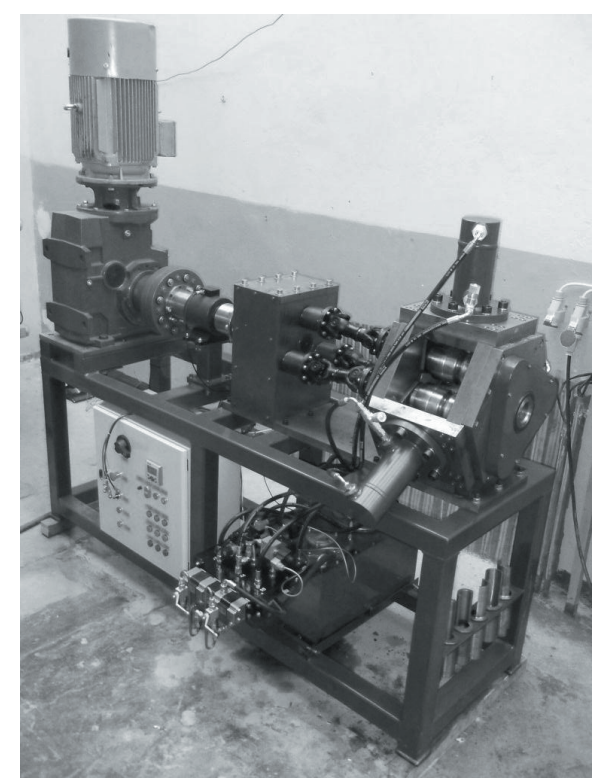

Fig. 5. Machine used in the experiments of the rotary compression process for hollow shafts 
The tubular specimens used in the experiments were $\mathrm{C} 45$ steel tubes of the same dimensions as those applied in the FEM simulation. The tubular specimens were first heated in an electrical chamber furnace to a forming temperature of approx. $1150{ }^{\circ} \mathrm{C}$, then they were fed by tongs into a special feed mechanism to position the tubular specimen in the working space of the machine (formed by three rotating rolls - Fig. 6a). In the subsequent stage of the process, the tools travelled radially at a constant velocity $v=2 \mathrm{~mm} / \mathrm{s}$ and were rotated in the same direction at a velocity $n=36$ rotations per minute, consequently setting the tubular specimen into a rotational motion and, thus, reducing the diameter of the step in the centre of the work piece. Once the slides travelled the path of the required diameter reduction $(\delta=D / d=1.5)$, the translational motion was automatically stopped. After the actual forming operation, the work piece was sized into the desired shape between rotating rolls. At the final stage of the process, the tools were opened radially (Fig. 6b) and the finished part was removed from the working space of the machine in a special feed mechanism.

The primary aim of the experiments was to verify whether the geometrical models applied in the numerical analysis were correct and to validate whether the rotary compression process is suitable for forming hollow stepped shafts. In the experiments of forming hollow tubular specimens with various wall thicknesses into shafts, we also analysed the geometrical parameters of finished products, process stability and forces. The experimental results obtained explicitly demonstrate that the proposed technique can be employed to form hollow tubular specimens into hollow stepped axes and shafts. The experimental results regarding the shapes and dimensions of produced parts agree well with both the theoretical assumptions (work piece shape assumed at design) and the numerical results. Fig. 7 shows the hollow shafts with various wall thicknesses produced in the experiments. As can be seen from Fig. 8, the thickness of the work piece walls increases, which is caused by the material's radial flow. Furthermore, it was observed that the length of all parts increased compared to the initial length of the tubular specimens, which results from the axial displacement of the material. It should however be stressed that the material flows axially mainly in the region where the tools have various radii, thus leading to a decrease in wall thickness in the region between the step being formed and the nondeformed steps of the work piece. The thinning of the wall can be significantly reduced or even eliminated by preventing free work piece elongation during the process.

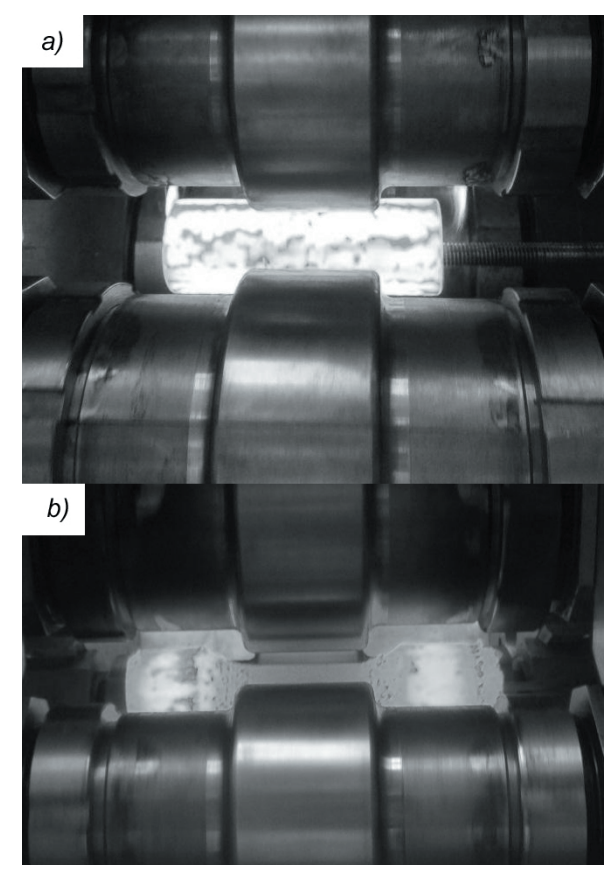

Fig. 6. Rotary compression process for producing hollow shafts; a) start of the process, and $b$ ) end of the process

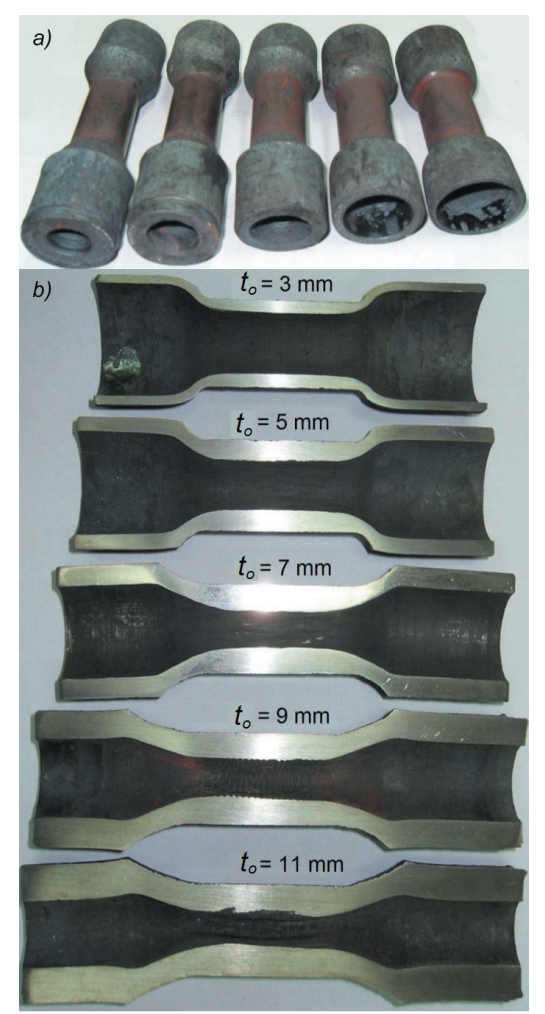

Fig. 7. Hollow shafts produced in the experiments from hollow tubular specimens with various wall thicknesses; a) general view, and b) axial sections 
In spite of the above, we can observe that the region between the end and central steps of the work piece is characterized by local thinning of the work piece wall. Apart from the local thinning of the work piece wall, a small arching of the axis of the shaft is noted, especially in regard to shafts formed from tubular specimens with thicker walls. This defect is caused by the lack of support for the work piece in the zone of the end steps that were not in contact with the tools during the process.

Examining the geometry of the produced parts (Fig. 7), it can be observed that the initial thickness of a tubular specimen wall has a significant effect on the kinematics of material flow during the rotary compression process. The reduction in the outer diameter leads to a change in the wall thickness and work piece length.

It is to be noted, however, that the relative increase in the wall thickness of the necking formed (compared to the initial thickness) decreases as the initial tubular specimen wall thickness increases (Fig. 8 ); what is more, in the case of the tubular specimen with the thickest wall $\left(t_{o}=11 \mathrm{~mm}\right)$, a small loss of
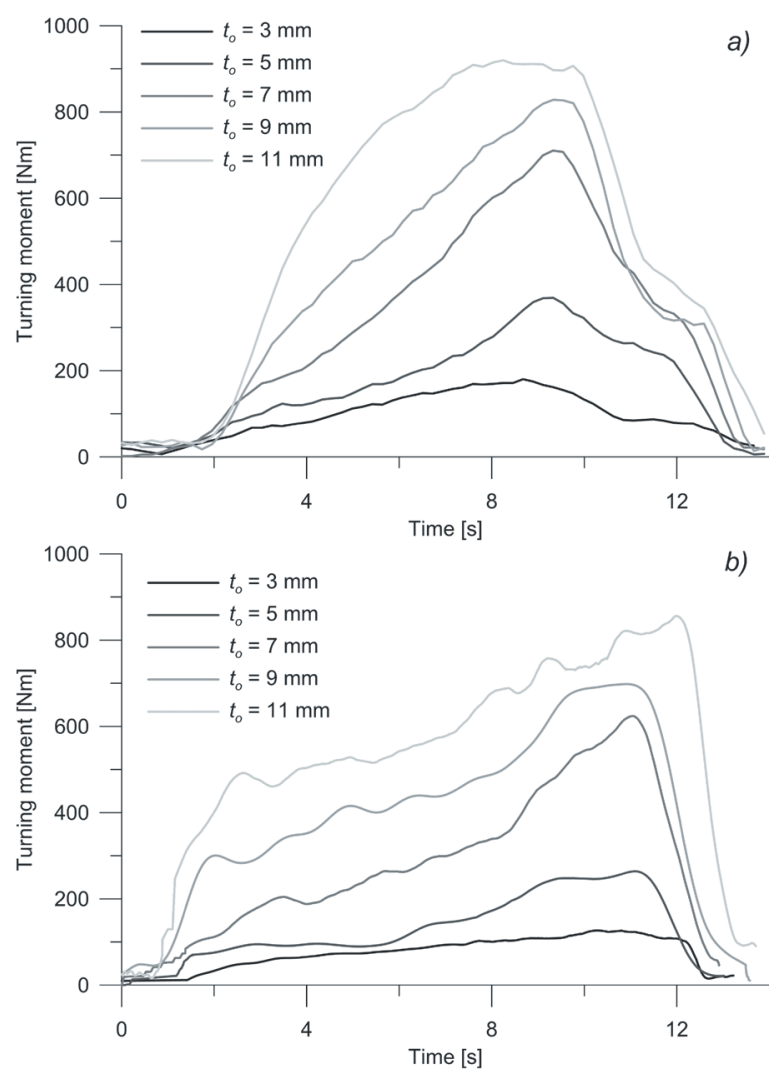

Fig. 9. Torque in the rotary compression for hollow shafts with various initial wall thicknesses; a) experiment, and b) FEM

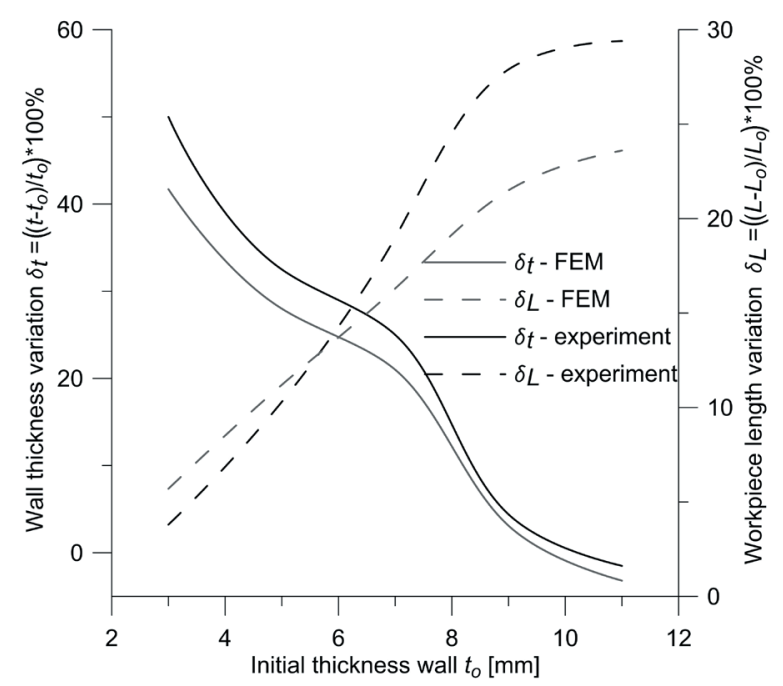

Fig. 8. Variations in the wall thickness and work piece length as determined by FEM and in the experiments

thickness of the wall in the formed step of the shaft can be observed. A reverse trend can be observed with regard to length of formed shafts. Here, the increase in the initial wall thickness leads to an increase in
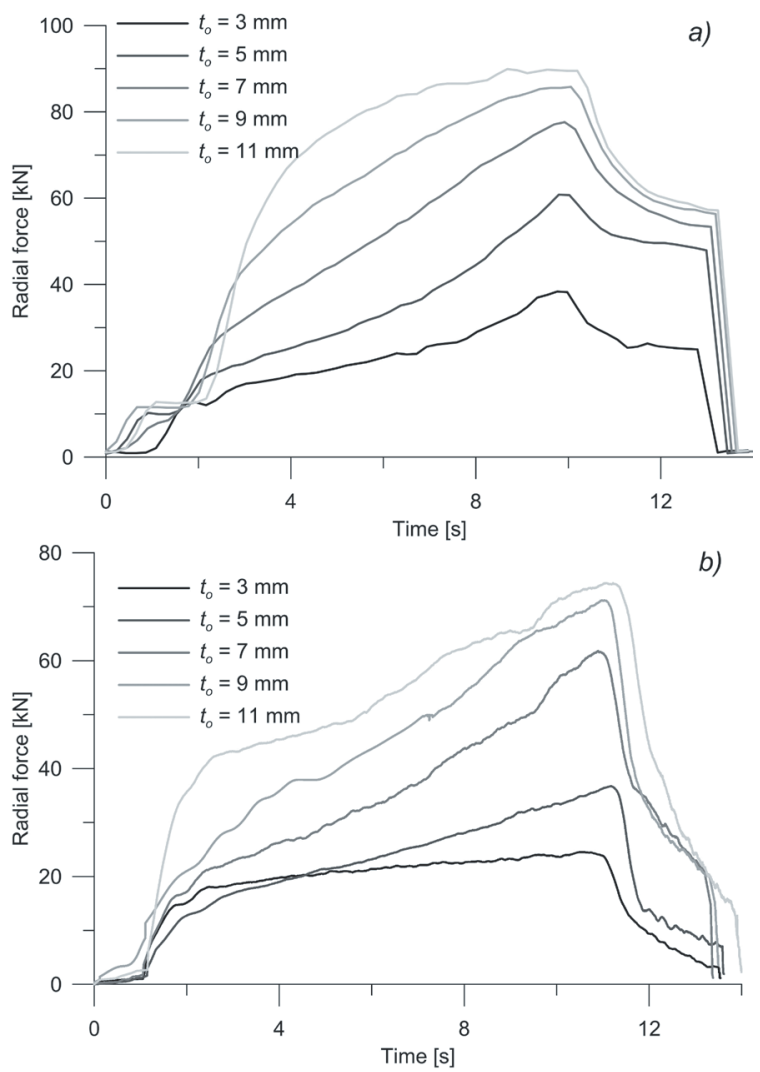

Fig. 10. Force in the rotary compression process for hollow parts with various initial wall thicknesses; a) experiment, and b) FEM 
length of the shaft. The observed relationship directly results from the differences in resistance to material flow that depend on tubular specimen wall thickness. In the forming of thin-walled tubular specimens, the resistances to the radial flow of the material are much lower than those observed when the material flow is axial, which leads to a rapid increase in the thickness of the walls of the shafts. With thicker walls, the material's axial flow is free from such resistances, which results in a rapid increase in the length of the shaft.

The theoretical and experimental analyses were also performed to investigate forces and torques of the process, the variations of which are shown in Figs. 9 and 10. It can be noted that an increase in the thickness of a tubular specimen wall leads to an increase in the forces and torques. The nature of the variations in the force parameters is similar in all investigated cases. Initially, the forces and torques increase rapidly with the sinking of the tools into the material; in the steady-state stage of the process, the increase in the forces is stable and gentle. Then, at the final stage of the process, a rapid decrease in the forces and torques can be observed. There are, however, differences between the FEM-calculated variations in the forces and torques and those obtained in the experiments. The experimental results demonstrate that the forces increase much faster over time compared to the variations of the forces obtained in the FEM modelling. Moreover, the forces and torques are higher in the experiments (the differences can even amount to several percentage points in the case of tubular specimens with thin walls). The observed differences can result from the fact that the material cools faster in the experiments than in the numerical modelling. This is proved by the considerably lower differences in values of the forces obtained in the forming of tubular specimens with thicker walls. Owing to the higher heat capacity of the tubular specimens, the temperature of the tubular specimen is relatively high, thus ensuring sufficient material workability.

\section{DISCUSSION AND CONCLUSIONS}

The majority of currently employed metal-forming techniques for hollow parts are highly complicated and relatively expensive; they only become costeffective in mass production. The proposed new rotary compression method for hollow parts can be successfully employed in both small series and mass production, as it is based on the use of simple tools and devices. Furthermore, the use of hollow tubular specimens in rotary compression leads to lower consumption of both material and energy, which, in turn, results in lower production costs.

The theoretical and experimental analyses of the rotary compression process have confirmed that stepped hollow shafts can be produced from hollow tubular specimens. The analyses led to a determination of the effect of work piece geometry on rotary compression and the geometry of finished parts. Furthermore, the force parameters of the process were investigated. Most importantly, however, the investigation helped validate the developed numerical model of the rotary compression process. Based on the results, the following conclusions have been drawn:

1. The numerical and experimental results show satisfactory agreement, which means that rotary compression processes can be modelled numerically.

2. Rotary compression can be employed to shape hollow tubular specimens of various wall thicknesses into stepped axes and shafts.

3. Parts produced by rotary compression processes exhibit higher wall thickness of steps and higher overall length of the work piece.

4. The rotary compression process for hollow parts involves non-uniform deformations; the greater the non-uniformity is, the higher the initial thickness of the tubular specimen wall is.

5. When tubular specimens with small initial wall thicknesses are formed into hollow parts, the material undergoes rapid cooling, which may hinder the rotary compression process.

6. An increase in the initial wall thickness of the tubular specimen leads to an increase in the tool force and torque.

7. Further research should be undertaken to determine failure modes and relationships between the technological parameters of the compression process and the geometry of tubular specimens and work piece.

\section{ACKNOWLEDGEMENTS}

The study was conducted as part of the project "Qualifications for the labour market - employer friendly university", co-financed by European Union from European Social Fund.

\section{REFERENCES}

[1] Opalić, M., Kranjčević, N., Habuš, S. (2011). Proof of strength of shafts and axles using finite element method. Transactions of FAMENA, vol. 35, no. 2, p. 63-71. 
[2] Neugebauer, R., Kolbe, M., Glass, R. (2001). New warm forming processes to produce hollow shafts. Journal of Materials Processing Technology, vol. 119, no. 1-3, p. $277-$ 282, D0I:10.1016/S09240136(01)00936.

[3] Neugebauer, R., Glass, R., Hoffmann, M. (2005). Spin Extrusion - A New Partial Forming Technology based on 7 NCAxes Machining. Annals of the CIRP, vol. 54, no 1, p. 241-244, DOl:10.1016/S0007-8506(07)60093-6.

[4] Stanić, I., Galeta, T., Karakašić, M. (2008). Optimization of protecting wall tool for manufacture and assembly. Technical Gazette, vol. 15, no. 1, p. 45-49.

[5] Pepelnjak, T. (2004). Numerical Analyses of Tube Hydroforming by High Internal Pressure. Strojniški vestnik - Journal of Mechanical Engineering, vol. 501, no. 1, p. 31-43.

[6] Staupendahl, D., Becker, C., Weinrich, A., Hermes, M., Tekkaya, A.E. (2012). Innovative forming processes for tubes, profiles and sheets made of modern steel grades. Stahl und Eisen, vol. 132, no. 8 p. 47-54.

[7] Jia, Z., Zhou, J., Ji, J.J., Yu, Y.-Y., Xiao, C. (2012). Influence of tool parameters on internal voids in cross wedge rolling of aluminum alloy parts. Transactions of Nonferrous Metals Society of China, vol. 22, suppl. 1, p. 21-26, D0l:10.1016/ S1003-6326(12)61678-1.

[8] Dadras, P., Wells, W.R. (1984). Heat transfer aspects of nonisothermal axisymmetric upset forging. Journal of Engineering for Industry, vol. 106, no. 3, p. 187-195, DOI:10.1115/1.3185932.

[9] Malinowski, Z., Lenard, J.G., Davies, M.E. (1994). A study of the heat-transfer coefficient as a function of temperature and pressure. Journal of Materials Processing Technology, vol. 41, no. 2, p. 125-142, Dol:10.1016/0924-0136(94)90057-4. 